\title{
Genetic diversity analysis of Bt cotton genotypes in Pakistan using simple sequence repeat markers
}

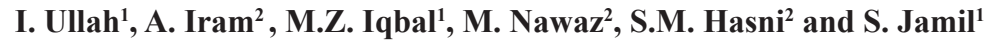 \\ ${ }^{1}$ Agricultural Biotechnology Research Institute, Faisalabad, Pakistan \\ ${ }^{2}$ Department of Bioinformatics and Biotechnology, GC University, \\ Faisalabad, Pakistan \\ Corresponding authors: I. Ullah \\ E-mail: ihsan.ullah_tlw@yahoo.com
}

Genet. Mol. Res. 11 (1): 597-605 (2012)

Received August 12, 2011

Accepted January 18, 2012

Published March 14, 2012

DOI http://dx.doi.org/10.4238/2012.March.14.3

\begin{abstract}
The popularity of genetically modified insect resistant (Bt) cotton has promoted large scale monocultures, which is thought to worsen the problem of crop genetic homogeneity. Information on genetic diversity among $\mathrm{Bt}$ cotton varieties is lacking. We evaluated genetic divergence among $19 \mathrm{Bt}$ cotton genotypes using simple sequence repeat (SSR) markers. Thirty-seven of 104 surveyed primers were found informative. Fifty-two primers selected on the basis of reported intra-hirsutum polymorphism in a cotton marker database showed a high degree of polymorphism, 56\% compared to $13 \%$ for randomly selected primers. A total of 177 loci were amplified, with an average of 1.57 loci per primer, generating 38 markers. The amplicons ranged in size from 98 to $256 \mathrm{bp}$. The genetic similarities among the 19 genotypes ranged from 0.902 to 0.982 , with an average of 0.947, revealing a lack of diversity. Similarities among genotypes from public sector organizations were higher than genotypes developed by private companies. Hybrids were found to be more distant compared to commercial cultivars and advanced breeding lines. Cluster analysis grouped the $19 \mathrm{Bt}$ cotton genotypes into three major clusters and two independent entries. Cultivars IR-3701, Ali Akbar-802 and advanced breeding line VH259 grouped in subcluster B2, with very narrow genetic distances despite
\end{abstract}


dissimilar parentage. We found a very high level of similarity among Pakistani-bred Bt cotton varieties, which means that genetically diverse recurrent parents should be included to enhance genetic diversity. The intra-hirsutum polymorphic SSRs were found to be highly informative for molecular genetic diversity studies in these cotton varieties.

Key words: SSRs; Bt cotton; Genetic diversity; Cotton marker database

\section{INTRODUCTION}

Cotton production is a substantial contributor to developing economies, including that of Pakistan, in which cotton encompasses more than half of the total exports (GOP, 2010). Enormous efforts from cotton breeders have improved the yield potential and fiber quality of modern cotton cultivars considerably. However, insect pests, primarily the bollworm complex, continuously threatened cotton productivity worldwide. The conventional pest-control approach of incorporating favorable plant characteristics into cotton cultivars has not imparted great degrees of host-plant resistance against insects.

Biotechnology has emerged as a potential tool for addressing problems that conventional research has not resolved. Among other applications of biotechnology, the development of genetically modified (GM) organisms is perhaps the most promising technique for facilitating plant breeding for the development of insect-resistant and herbicide-tolerant crop cultivars. The development and commercial release of the first genetically engineered insect resistant cotton cultivar Bollgard $^{\mathbb{Q}}$, which expressed Cry1Ac $\delta$-endotoxin from Bacillus thuringiensis $(\mathrm{Bt})$ var kurstaki strain HD-1 (Perlak et al., 1990), opened a new arena in this field. Insect-resistant cotton, commonly referred to as Bt cotton, has shown resistance to important lepidopterous cotton pests in the laboratory as well as in the field (Fitt et al., 1994; Halcomb et al., 1996; Tabashnik et al., 2008). Second-generation GM cottons were released that contained multiple genes for insect resistance and herbicide tolerance. The effectiveness of Bt cotton varieties in the control of bollworms makes it a first choice in farming communities. Its popularity can be gauged from the many-fold increase in areas under cultivation with these varieties during the last 15 years. In 2010, more than 21 million hectares were planted with GM cottons in six major cotton-growing countries (James, 2010).

In Pakistan, exotic Bt cotton varieties entered cultivation through informal channels. Later, breeders from the public and private sectors developed Bt varieties with local genetic backgrounds through backcrossing with exotic Bt cotton containing the Cry1 Ac gene (MON531 event). As in other parts of the world, the adoption of Bt technology in Pakistan is unprecedented: in 2010, approximately 600,000 farmers had planted Bt cotton (James, 2010).

Regeneration response in cotton is highly genotype specific (Trolinder and Xhixian, 1989). Most first- and second-generation GM cottons, including Bollgard ${ }^{\mathbb{B}}$, were developed through the incorporation of transgenes in regeneration-responsive genotypes and then transferred to the desirable genetic background through conventional means. Availability of limited regeneration-responsive genotypes and the use of selective varieties as recurrent parents in the development of GM cotton cultivars has reinforced genetic homogeneity and promoted largescale monocultures, which may contribute to increased vulnerability to biotic and abiotic stresses.

The development of DNA markers has expedited efforts to investigate genetic variability, parentage assignments, varietal identification, and the construction of high-resolution genetic linkage 
maps in different crop species. Among the wide array of DNA marker systems available, microsatellite markers, also known as simple sequence repeats (SSRs), have emerged as the markers of choice for genetic assay. Approximately 17,448 cotton SSR primer pair sequences are available in the public domain (http://www.cottonmarker.org/Primer.shtml; accessed January 9, 2012). The availability of such a large number of SSRs has opened new avenues for genetic and genomic studies in cotton, including hybrid identification (Dongre and Parkhi, 2005) and genetic diversity estimation among diploid (Rahman et al., 2008) and tetraploid (Lacape et al., 2007; Wang et al., 2007) germplasms and commercial cultivars (Gutiérrez et al., 2002; Zhang et al., 2005; Bertini et al., 2006). However, no studies have been conducted to estimate genetic relatedness among Bt cottons. Hence, this study aimed to use SSR markers to explore genetic diversity among 19 Bt cotton genotypes from Pakistan, including eight commercial cultivars, two hybrids, and nine advanced breeding lines.

\section{MATERIAL AND METHODS}

\section{Plant material}

Nineteen Bt cotton genotypes comprising eight commercial cultivars, two hybrids, and nine advanced breeding lines were evaluated in this study (hereafter referred to as genotypes). The tested insect-resistant transgenic cotton material, except that of the two hybrids, contained the Cry1Ac gene from MON531 transformation event, incorporated through backcrossing. A list of genotypes, their origins, and pedigree information is provided in Table 1.

Table 1. Name, pedigrees and origin of eight insect resistant cotton commercial cultivars (bold), two hybrids (italic) and nine advanced breeding lines used to estimate genetic distance.

\begin{tabular}{lll}
\hline Name & Origin & Pedigree \\
\hline Ali Akbar-802 & Ali Akbar Seeds, Pakistan & FH-1000/**HK-303/LRA-5166/LINEA-100 \\
Ali Akbar-703 & Ali Akbar Seeds, Pakistan & CIM-482/**HK-303 \\
Sitara-008 & Aziz Group, Pakistan & Confidential \\
BT-473 & Central Cotton Research Institute, Multan & Confidential \\
BT-886 & Cotton Research Station, Multan & FH-207/MNH-6070/*Bollgard \\
FH-113 & Cotton Research Institute, Faisalabad & CIM-448/*Bollgard \\
$G N-2085$ & Nath Seeds, India & Confidential \\
GN-31 & Nath Seeds, India & Confidential \\
IR-1524 & National Institute for Biotechnology \& & NIBGE-2/*Transgenic Bt cotton line \\
& Genetic Engineering (NIBGE), Faisalabad & \\
IR-2381 & NIBGE, Faisalabad & FH-1000/*Transgenic Bt cotton line \\
IR-2389 & NIBGE, Faisalabad & Selection from IR-2381 \\
IR-3701 & NIBGE, Faisalabad & CIM-448/*Transgenic Bt cotton line \\
IR-901 & NIBGE, Faisalabad & FH-901/*Transgenic Bt cotton line \\
MG-6 & Cotton Research Station, Multan & CIM-443/**IR-448 \\
Neelum-121 & Neelum Seeds, Vehari & A9-17/*EXOTIC-1 \\
VH-255 & Cotton Research Station, Vehari & VH-137/**IR-901 \\
VH-259 & Cotton Research Station Vehari & CIM-511/**Neeelum-121 \\
VH-279 & Cotton Research Station Vehari & VH-148/**Neelum-121 \\
VH-296 & Cotton Research Station Vehari & Confidential \\
\hline
\end{tabular}

*Primary exotic transgenic source for the Cry1Ac insect resistance gene (MON531 event). **Secondary transgenic source for the Cry1Ac insect resistance gene (MON531 event).

\section{DNA extraction}

Total genomic DNA was isolated from bulked leaves obtained from 3-4 randomly se- 
lected plants of each genotype using the cetyltrimethylammonium bromide method (Iqbal et al., 1997) with minor modifications. The quantification of isolated DNA was conducted using a spectrophotometer (NanoDrop-2000, Thermo Scientific, USA), and quality was evaluated by loading 50 ng DNA from each genotype on a $0.8 \%$ agarose gel.

\section{SSR markers}

One hundred and four cotton SSR primer pairs representing at least two markers from each chromosome were selected for genotyping. The Cotton Marker Database (CMD; http:// www.cottonmarker.org) was explored to select primers and download their sequences. Half of the total primers used were selected on the basis of intra-hirsutum polymorphism provided in panel information in the database.

\section{SSR analysis}

For SSR analysis, a reaction volume of $20 \mu \mathrm{L}$ was made containing $2.0 \mu \mathrm{L} 10 \mathrm{X}$ polymerase chain reaction buffer (50 mM Tris, $\mathrm{pH} 8.3,500 \mathrm{mM} \mathrm{KCl}) ; 1.5 \mathrm{mM} \mathrm{MgCl} ; 0.2 \mathrm{mM}$ each of dATP, dCTP, dGTP, and dTTP (Fermentas, USA); $0.6 \mu \mathrm{M}$ each of reverse and forward primer (GeneLink, USA); 1 U Taq DNA polymerase (Fermentas), and $50 \mathrm{ng}$ genomic DNA as a template. Amplification was performed in a Mastercycler Gradient (Eppendorf, Germany) with the following temperature cycles: first denaturation step of $94^{\circ} \mathrm{C}$ for 5 min followed by 35 cycles of $30 \mathrm{~s}$ at $94^{\circ} \mathrm{C}, 30 \mathrm{~s}$ at approximately $55^{\circ} \mathrm{C}$ (varied with the melting temperature of the primers), and $1 \mathrm{~min}$ at $72^{\circ} \mathrm{C}$. A final extension was performed at $72^{\circ} \mathrm{C}$ for $5 \mathrm{~min}$. Amplified products were resolved on 6\% polyacrylamide gel (19:1, acryl:bis) on an OmniPAGE Maxi Vertical Electrophoresis system (BiocomDirect, UK) and visualized with silver staining.

\section{Data analysis}

To analyze the genetic relationships among the $19 \mathrm{Bt}$ cotton genotypes assayed, we considered each band a single locus/allele. Genetic similarity matrices were generated based on Nei's coefficients (Nei, 1972). NTSyspc 2.0 was used for dendrogram construction with the unweighted-pair group method of arithmetic means (UPGMA).

\section{RESULTS}

One hundred and four SSR primer pairs, 76 from the Brookhaven National Laboratory (BNL) series and 28 from the Centre International en Recherche Agronomique pour le Développement (CIR) series, were surveyed to evaluate genetic diversity among $19 \mathrm{Bt}$ cotton genotypes from Pakistan (eight commercial cultivars, two hybrids, and nine advanced breeding lines). The CMD was used to select intra-hirsutum informative SSR primer pairs. Exploration of the BNL and CIR panel screen data in the database revealed 145 and 62 polymorphic primers, respectively, among six Gossypium hirsutum (AD1) lines (TM-1, Acala Maxxa, DPL 458BR, Paymaster 1218BR, Fibermax 832, and Stoneville 4892BR). From these 207 intra-hirsutum informative primers, 52 markers, preferably those amplifying single loci and loci $\leq 256 \mathrm{bp}$ in size, were selected for the study. An additional 52 primers were picked randomly, representing at least one marker from each chromosome. 
Thirty-seven of the 104 surveyed primers were found informative. Among 52 primers selected on the basis of reported intra-hirsutum polymorphism in the CMD database, 30 were found informative with a polymorphism rate of $56 \%$, whereas the rate of informative primers from the 52 randomly selected SSR primers was $13 \%$. Where series-wise polymorphism was concerned, the BNL series showed a high level of polymorphism (47\%) compared to that of the CIR series, in which only two primers were found informative. A total of 177 loci were amplified with an average of 1.57 loci per primer, generating 38 markers. The amplified DNA fragments ranged from 98 to $256 \mathrm{bp}$. A maximum of three loci were amplified by the SSR primer BNL 3545. The surveyed SSR primers produced dominant as well as co-dominant polymorphism; however, the proportion of dominant markers was relatively low compared with that of co-dominant markers. Only two of 38 markers behaved dominantly.

Data obtained from SSR analysis was used to generate a similarity matrix using the method of Nei (1972) with data from 38 markers. The resulting matrix showed a mean genetic similarity of 0.947 among the 19 genotypes, revealing a very high level of genetic relatedness. Where pairwise combinations were concerned, genetic similarities between genotypes varied from 0.902 to 0.982 (Table 2). Cultivar Neelum-121 and hybrid GN-31 were the most diverse, with a genetic distance of $10 \%$. The cultivar IR-3701 showed a high similarity of 0.982 with the cultivar Ali Akbar-802 and advanced breeding line VH-259. Similarly, the cultivar IR-1524 and advanced breeding line VH-279 were highly related, with a similarity coefficient of 0.983 .

Table 2. Genetic similarity matrix of eight insect-resistant cotton commercial cultivars (bold), two hybrids (italic) and nine advanced breeding lines using 38 simple sequence repeat marker loci.

\begin{tabular}{|c|c|c|c|c|c|c|c|c|c|c|c|c|c|c|c|c|c|c|}
\hline Accession & $\begin{array}{c}\text { IR- } \\
2389\end{array}$ & $\begin{array}{c}G N- \\
31\end{array}$ & $\begin{array}{l}\text { IR- } \\
2381\end{array}$ & $\begin{array}{l}\text { BT- } \\
886\end{array}$ & $\begin{array}{l}\text { VH- } \\
255\end{array}$ & $\begin{array}{l}\text { VH- } \\
259\end{array}$ & $\begin{array}{c}\text { MG- } \\
6\end{array}$ & $\begin{array}{l}\text { VH- } \\
296\end{array}$ & $\begin{array}{l}\text { IR- } \\
901\end{array}$ & $\begin{array}{l}\text { VH- } \\
279\end{array}$ & $\begin{array}{l}\text { BT- } \\
473\end{array}$ & $\begin{array}{c}\text { Sitara- } \\
008\end{array}$ & $\begin{array}{l}\text { IR- } \\
3701\end{array}$ & $\begin{array}{l}\text { IR- } \\
1524\end{array}$ & $\begin{array}{l}\text { A. Akbar- } \\
802\end{array}$ & $\begin{array}{l}\text { A. Akbar- } \\
703\end{array}$ & $\begin{array}{c}\text { Neelum- } \\
121\end{array}$ & $\begin{array}{r}-G N- \\
2085\end{array}$ \\
\hline$G N-31$ & 0.935 & & & & & & & & & & & & & & & & & \\
\hline IR-2381 & 0.970 & 0.931 & & & & & & & & & & & & & & & & \\
\hline BT-886 & 0.956 & 0.928 & 0.951 & & & & & & & & & & & & & & & \\
\hline VH-255 & 0.956 & 0.940 & 0.951 & 0.949 & & & & & & & & & & & & & & \\
\hline VH-259 & 0.937 & 0.922 & 0.957 & 0.956 & 0.943 & & & & & & & & & & & & & \\
\hline MG-6 & 0.923 & 0.934 & 0.957 & 0.929 & 0.942 & 0.949 & & & & & & & & & & & & \\
\hline VH-296 & 0.930 & 0.955 & 0.951 & 0.962 & 0.949 & 0.969 & 0.942 & & & & & & & & & & & \\
\hline IR-901 & 0.936 & 0.920 & 0.970 & 0.916 & 0.955 & 0.949 & 0.962 & 0.929 & & & & & & & & & & \\
\hline VH-279 & 0.937 & 0.935 & 0.957 & 0.956 & 0.943 & 0.975 & 0.949 & 0.969 & 0.936 & & & & & & & & & \\
\hline BT-473 & 0.930 & 0.941 & 0.951 & 0.949 & 0.962 & 0.956 & 0.929 & 0.962 & 0.956 & 0.956 & & & & & & & & \\
\hline Sitara-008 & 0.956 & 0.927 & 0.951 & 0.962 & 0.935 & 0.943 & 0.942 & 0.949 & 0.928 & 0.929 & 0.922 & & & & & & & \\
\hline IR-3701 & 0.957 & 0.944 & 0.976 & 0.963 & 0.951 & 0.982 & 0.957 & 0.976 & 0.957 & 0.970 & 0.963 & 0.963 & & & & & & \\
\hline IR-1524 & 0.944 & 0.929 & 0.964 & 0.963 & 0.937 & 0.957 & 0.943 & 0.950 & 0.943 & 0.982 & 0.963 & 0.923 & 0.964 & & & & & \\
\hline A. Akbar-802 & 0.950 & 0.935 & 0.970 & 0.943 & 0.956 & 0.975 & 0.962 & 0.956 & 0.975 & 0.963 & 0.956 & 0.943 & 0.982 & 0.969 & & & & \\
\hline A. Akbar-703 & 0.950 & 0.935 & 0.970 & 0.930 & 0.956 & 0.963 & 0.962 & 0.943 & 0.975 & 0.937 & 0.956 & 0.956 & 0.970 & 0.931 & 0.963 & & & \\
\hline Neelum-121 & 0.931 & 0.902 & 0.964 & 0.937 & 0.937 & 0.944 & 0.943 & 0.937 & 0.943 & 0.944 & 0.937 & 0.923 & 0.951 & 0.951 & 0.944 & 0.957 & & \\
\hline$G N-2085$ & 0.936 & 0.919 & 0.918 & 0.955 & 0.941 & 0.936 & 0.921 & 0.928 & 0.921 & 0.949 & 0.942 & 0.941 & 0.944 & 0.943 & 0.922 & 0.936 & 0.930 & \\
\hline FH-113 & 0.924 & 0.922 & 0.957 & 0.943 & 0.929 & 0.924 & 0.949 & 0.943 & 0.949 & 0.937 & 0.943 & 0.929 & 0.945 & 0.944 & 0.924 & 0.950 & 0.969 & 0.949 \\
\hline
\end{tabular}

Similarity coefficients among genotypes from public-sector research organizations were higher than those developed by private seed companies (Table 3). Among public-sector organization genotypes, those from the Cotton Research Station Multan were relatively diverse (0.929) compared to those of the National Institute for Biotechnology \& Genetic Engineering (NIBGE), Faisalabad, and the Cotton Research Station, Vehari, for which the average similarity coefficient was 0.958 . Among private-sector seed companies, material from Nath Seeds, India, showed a comparatively lower mean similarity of 0.919 . Comparison of the type of material revealed that hybrids were more distinct than commercial cultivars and advanced 
breeding lines (see Table 3). Commercial cultivars and pipeline material had mean similarity coefficients of 0.950 and 0.951 , respectively.

\begin{tabular}{llr} 
Table 3. Range and mean genetic similarity within Bt cotton genotypes of different source/type. \\
\hline Genotype source/type & \multicolumn{1}{c}{ Similarity coefficient } \\
\cline { 2 - 3 } & \multicolumn{1}{c}{ Range } & Mean \\
\hline All 19 genotypes & $0.902-0.982$ & 0.947 \\
Genotypes from public sector organizations & $0.923-0.982$ & 0.951 \\
National Institute for Biotechnology \& Genetic & $0.936-0.976$ & 0.958 \\
Engineering, Faisalabad & $0.943-0.975$ & 0.958 \\
Cotton Research Station, Vehari & 0.929 & 0.929 \\
Cotton Research Station, Multan & $0.902-0.963$ & 0.935 \\
Genotypes from private sector & 0.919 & 0.919 \\
Nath Seeds, India & 0.963 & 0.963 \\
Ali Akbar Seeds, Pakistan & $0.923-0.982$ & 0.950 \\
Eight commercial cultivars & $0.916-0.975$ & 0.951 \\
Eleven advanced breeding lines & 0.919 & 0.919 \\
Two hybrids & &
\end{tabular}

The UPGMA tree generated from genetic distance coefficients grouped the $19 \mathrm{Bt}$ cotton genotypes into three major clusters and two independent entries (Figure 1). Cluster A contained two subclusters, A1 (IR-2389 and IR-2381) and A2 (BT-886 and Sitara-008). Cluster B grouped eight genotypes into three subgroups, in which B1 contained VH-255 and Bt473. The genotypes VH-259, IR-3701, Ali Akbar-802, VH-296, VH-279, and IR-1524 were included in subcluster B2. Subcluster B3 comprised the cultivars MG-6, Ali Akbar-703, and advanced breeding line IR-901. The cultivars Neelum-121 and FH113 were grouped in cluster C, whereas the Bt hybrids GN-2085 and GN-31 remained distinct from the other genotypes.

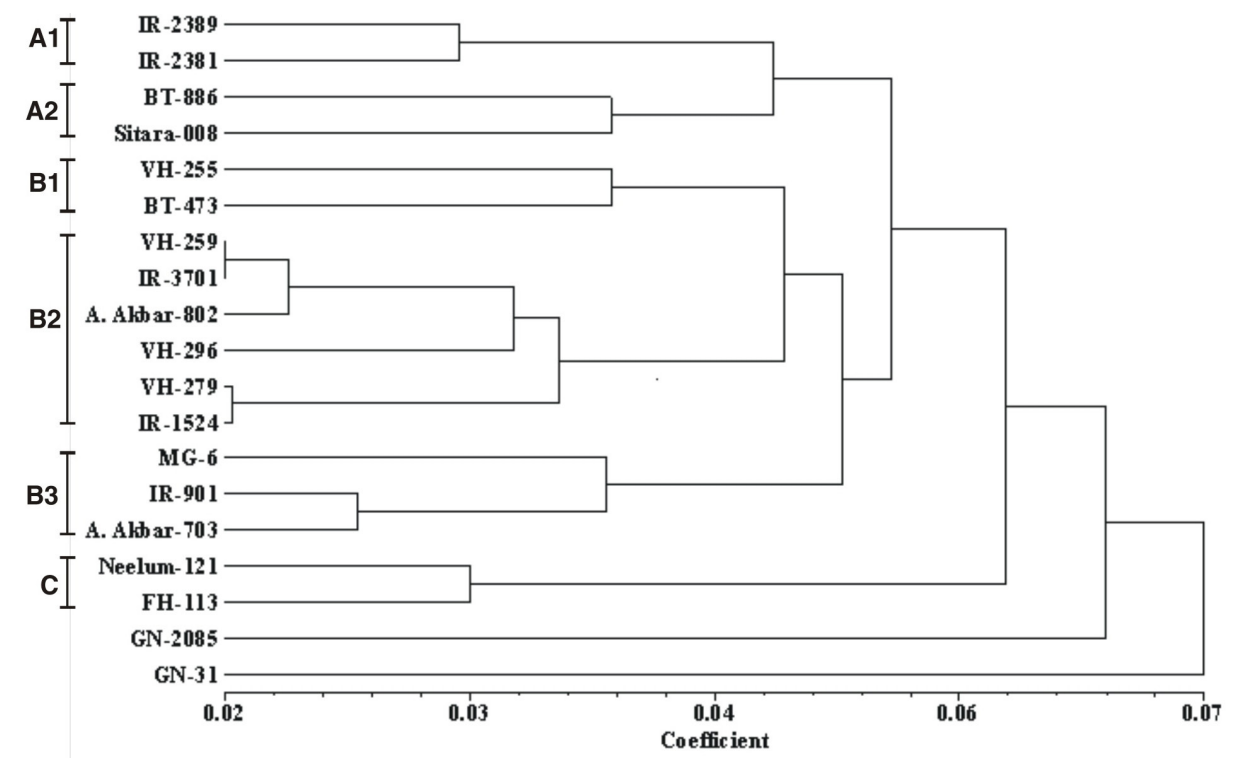

Figure 1. Dendogram presenting the association of 19 insect-resistant cotton genotypes generated by unweighted paired group method by arithmetic averages (UPGMA) method using 38 simple sequence repeat marker loci. 


\section{DISCUSSION}

Genetic diversity in germplasm is a prerequisite for coping with environmental changes and is typically described using polymorphism. Microsatellites are the markers of choice for genetic diversity analysis owing to a high polymorphism rate. The reported percentage of polymorphic SSR primers is low in cotton; however, necessitating the application of a relatively higher number of primers to obtain considerable information for genetic distance estimates (Rahman et al., 2002). Application of known polymorphic primers is helpful for increasing the amount of information available (Zhang et al., 2005; Lacape et al., 2007). Results from this study indicate that the information about intra-hirsutum polymorphic SSR primers provided in the CMD is highly useful for primer selection to obtain a high percentage of polymorphism in upland cottons.

The results of genetic distance estimates obtained in our study revealed a very high similarity of 90.2 to $98.2 \%$ among the 19 genotypes studied. Low genetic diversity estimates have also been reported in cotton using different DNA marker systems. For nine Australianbred cotton cultivars, genetic distance coefficients ranged from 0.01 to 0.08 (Multani and Lyon, 1995). Similarly, Lu and Myers (2002) reported genetic diversity of 2 to 7\% using amplified polymorphic DNA markers with 10 influential U.S. cotton germplasm lines. Limited genetic variation has also been reported in cotton by several other researchers (Lacape et al., 2007; Wang et al., 2007; Rahman et al., 2008). We found a mean genetic similarity of 0.947 among the 19 Pakistani-bred Bt cotton genotypes examined, revealing very high level of genetic relatedness. Although no previous studies have been conducted on the genetic diversity of Pakistani-bred Bt cotton, previous findings on genetic diversity analysis of Pakistani-bred non-Bt cotton have also revealed low estimates for genetic distances (Iqbal et al., 1997, 2001; Rahman et al., 2002) with the exception of Khan et al. (2009), who reported a medium mean similarity of $75 \%$. This finding may be attributed to the relatively large number of accessions used in the study, which comprised 40 representative cotton cultivars released from 1914 to 2005.

Similarity within cultivars and within advanced breeding lines was generally higher than that among hybrids (see Table 3), which was an expected finding. Genotypes developed by public-sector organizations were fairly similar compared to those produced in the private sector. Among public-sector organization genotypes, those from NIBGE were less diverse compared to those from the Cotton Research Station, Multan (see Table 3). These relationships are also supported by pedigree information of the genotypes from NIBGE, at which cotton cultivars FH-1000 and CIM-448 have been used as recurrent parents.

The monoculture of a few successful cultivars has promoted genetic uniformity in cotton (Van Esbroeck et al., 1998). Modern cotton cultivars have been bred through repeated hybridization of closely related parents, selection for better fiber quality and yield, and reselection within cultivars, leading to additional similarity (Bowman, 2000). The status of Pakistani-bred cotton is even more uniform. The recent breeding history of Pakistani cottons may shed light on this high uniformity. Most of the cotton material bred until the early 1990s was produced from crosses of local varieties with American cotton germplasm. That material was relatively diverse (Rahman et al., 2002). In 1992, however, an epidemic of cotton leaf curl virus disease $(\mathrm{CLCuD})$ appeared. This menace played havoc with Pakistani cotton, and total productivity was considerably reduced. All cultivars and germplasm lines except LRA5166, CP15/2, and CEDIX proved to be susceptible to this new disease. Post-CLCuD era cotton varieties were then developed using one of the three resistant lines to incorporate resistance against the dis- 
ease. As a result, the already narrow genetic base narrowed further (Rahman et al., 2002). In 1998, the exotic Bt variety Bollgard ${ }^{\circledR}$, containing the Cry1Ac insect-resistance gene, was introduced through informal sources in Pakistan. Later, Bt varieties were developed with local genetic backgrounds by crossing Bollgard ${ }^{\circledR}$ with local germplasms. All genotypes included in this study except hybrids GN-2085 and GN-31 were developed using either Bollgard ${ }^{\circledR}$ or its derivatives as donor parents, leading to very high similarity among the present pool of Bt varieties and advanced breeding lines. Our results indicate a very high level of similarity among Pakistani-bred Bt cottons. This lack of diversity may make them vulnerable to potential epidemics such as CLCuD, which necessitates the induction of diverse recurrent parents to enhance genetic diversity. Intra-hirsutum polymorphic SSRs provided on the CMD are highly informative for molecular genetic diversity studies in cotton, especially for resourcepoor laboratories with limited funding in developing countries.

\section{REFERENCES}

Bertini CHCM, Schuster I, Sediyama T, Barros EG, et al. (2006). Characterization and genetic diversity analysis of cotton cultivars using microsatellites. Genet. Mol. Biol. 29: 321-329.

Bowman DT (2000). Attributes of public and private cotton breeding programs. J. Cotton Sci. 4: 130-136.

Dongre A and Parkhi V (2005). Identification of cotton hybrid through the combination of PCR based RAPD, ISSR and microsatellite markers. J. Plant Biochem. Biot. 14: 53-55.

Fitt GP, Mares CL and Llewellyn DJ (1994). Field evaluation and potential ecological impact of transgenic cottons (Gossypium hirsutum) in Australia. Biocontrol. Sci. Tech. 4: 535-548.

GOP (2010). Economic Survey of Pakistan: Finance Division. Government of Pakistan, Pakistan.

Gutiérrez OA, Basu S, Saha S, Jenkins JN, et al. (2002). Genetic distance among selected cotton genotypes and its relationship with F2 performance. Crop Sci. 42: 1841-1847.

Halcomb JL, Benedict JB, Cook B and Ring DR (1996). Survival and growth of bollworm and tobacco budworm on nontransgenic and transgenic cotton expressing a CryIA insecticidal protein (Lepidoptera: Noctuidae). Environ. Entomol. 25: 250-255.

Iqbal MJ, Aziz N, Saeed NA, Zafar Y, et al. (1997). Genetic diversity evaluation of some elite cotton varieties by RAPD analysis. Theor. Appl. Genet. 94: 139-144.

Iqbal MJ, Reddy OUK, El-Zak KM and Pepper AE (2001). A genetic bottleneck in the "evolution under domestication" of upland cotton Gossypium hirsutum L. examined using DNA fingerprinting. Theor. Appl. Genet. 103: 547-554.

James C (2010). Global Status of Commercialized Biotech/GM Crops. 2010 ISAAA Brief No. 42. ISAAA, Ithaca.

Khan AI, Fu YB and Khan IA (2009). Genetic diversity of Pakistani cotton cultivars as revealed by simple sequence repeat markers. CBCS 4: 21-30.

Lacape JM, Dessauw D, Rajab M, Noyer JL, et al. (2007). Microsatellite diversity in tetraploid Gossypium germplasm: Assembling a highly informative genotyping set of cotton SSRs. Mol. Breed. 19: 45-58.

$\mathrm{Lu} \mathrm{J}$ and Myers O (2002). Genetic relationships and discrimination of ten influential upland cotton varieties using RAPD markers. Theor. Appl. Genet. 105: 325-331.

Multani DS and Lyon BR (1995). Genetic fingerprinting of Australian cotton cultivars with RAPD markers. Genome 38: 1005-1008.

Nei M (1972). Genetic distance between populations. Am. Nat. 106: 283-292.

Perlak FJ, Deaton RW, Armstrong TA, Fuchs RL, et al. (1990). Insect resistant cotton plants. Biotechnology 8: 939-943.

Rahman M, Hussain D and Zafar Y (2002). Estimation of genetic divergence among elite cotton cultivars-genotypes by DNA fingerprinting technology. Crop Sci. 42: 2137-2144.

Rahman M, Yasmin T, Tabassum N, Ullah I, et al. (2008). Studying the extent of genetic diversity among Gossypium arboreum L. genotypes/cultivars using DNA fingerprinting. Genet. Resour. Crop Evol. 55: 331-339.

Tabashnik BE, Gassmann AJ, Crowder DW and Carriere Y (2008). Insect resistance to Bt crops: evidence versus theory. Nat. Biotechnol. 26: 199-202.

Trolinder NL and Xhixian C (1989). Genotype specificity of the somatic embryogenesis response in cotton. Plant Cell Rep. 8: 133-136.

Van Esbroeck GA, Bowman DT, Calhoun DS and May OL (1998). Changes in the genetic diversity of cotton in the USA 
from 1970 to 1995. Crop Sci. 38: 33-37.

Wang X, Ma J, Yang S, Zhang G, et al. (2007). Assessment of genetic diversity among Chinese Upland cottons with Fusarium and/or Verticillium wilts resistance by AFLP and SSR markers. Front Agric. China. 1: 129-135.

Zhang J, Lu Y, Cantrell RG and Hughs E (2005). Molecular marker diversity and field performance in commercial cotton cultivars evaluated in the southwestern USA. Crop Sci. 45: 1483-1490. 